\title{
Snyder Space-Time: K-Loop and Lie Triple System ${ }^{\star}$
}

\section{Florian GIRELLI}

\author{
School of Physics, The University of Sydney, Sydney, New South Wales 2006, Australia \\ E-mail: girelli@physics.usyd.edu.au
}

Received April 29, 2010, in final form September 13, 2010; Published online September 24, 2010 doi:10.3842/SIGMA.2010.074

\begin{abstract}
Different deformations of the Poincaré symmetries have been identified for various non-commutative spaces (e.g. $\kappa$-Minkowski, $\mathfrak{s l}(2, R)$, Moyal). We present here the deformation of the Poincaré symmetries related to Snyder space-time. The notions of smooth "K-loop", a non-associative generalization of Abelian Lie groups, and its infinitesimal counterpart given by the Lie triple system are the key objects in the construction.
\end{abstract}

Key words: Snyder space-time; quantum group

2010 Mathematics Subject Classification: 17C90; 81T75

\section{Introduction}

Snyder space-time has been introduced in 1947, and is one of the first examples of non-commutative geometry [1]. The physical features of a quantum field theory living on this space are not very well known since there have been very few attempts to construct such field theory on this space (see [2] and references therein). It is only recently that a star product and a scalar field theory have been constructed $[3,4]$. Using a different route and doing the analysis at first order, [5] reached similar results. In both of these works, the scalar field action is shown to be invariant under a new type of deformed Poincaré symmetries. The main feature of this deformed symmetry is the non-(co-)associativity, which explains why this deformation did not appear when considering the (co-)associative deformations of the Poincaré group [6]. In this perspective, the natural question to ask is what is the algebraic structure or "quantum group" encoding the deformation of the Poincaré symmetries consistent with the Snyder non-commutativity? We present here the answer to this question by defining a new type of quantum group.

Before explaining the strategy we shall use in the Snyder case, let us recall the construction in the $\kappa$-Minkowski non-commutative space which is well understood and can serve as a guiding line. In this case, the relevant deformation of the Poincaré symmetries, the $\kappa$-Poincaré deformation [7, 8], is based on the Iwasawa decomposition of $\mathrm{SO}(p, 1) \sim \operatorname{AN}_{p} \cdot \operatorname{SO}(p-1,1) \sim$ $\mathrm{AN}_{p} \bowtie \mathrm{SO}(p-1,1)[9,10]$. The (Abelian nilpotent) group $\mathrm{AN}_{p}$ is interpreted as momentum space when dealing with a scalar field theory living in $\kappa$-Minkowski. The deformation of the translation symmetry can be traced back to the non-Abelian $\mathrm{AN}_{p}$ group structure. The $\kappa$-Poincaré (or bicrossproduct) algebra is encoded by $\mathcal{B}=\mathcal{C}\left(\mathrm{AN}_{p}\right) \triangleleft k \mathrm{SO}(p-1,1)$, where the algebra of functions over $\mathrm{AN}_{p}$ coacts on the group algebra $k \mathrm{SO}(p-1,1)$, and $k \mathrm{SO}(p-1,1)$ acts on $\mathrm{AN}_{p}$. This quantum group encodes the deformation of the Poincaré Hopf algebra $\mathcal{C}\left(\mathbb{R}^{p}\right) \rtimes k \mathrm{SO}(p-$ $1,1) \stackrel{\kappa}{\longrightarrow} \mathcal{C}\left(\mathrm{AN}_{p}\right) \triangleleft k \mathrm{SO}(p-1,1)$, by implementing the Planck scale $\kappa$. The $\kappa$-Minkowski space is encoded into the coordinate operators $X_{\mu}$ which satisfy the same commutator relations as the Lie algebra $\mathfrak{a n}_{p}$ of $\mathrm{AN}_{p}$. Using the Weyl map, it is possible to construct a *-product between c-number coordinates $x_{\mu}$ which satisfy the same commutation relations as $\mathfrak{a n}_{p}$ [11].

\footnotetext{
*This paper is a contribution to the Special Issue "Noncommutative Spaces and Fields". The full collection is available at http://www.emis.de/journals/SIGMA/noncommutative.html
} 
We can follow a similar route to construct the relevant non-commutative space associated to Snyder space-time. The Snyder deformation will be based on the decomposition $\operatorname{SO}(p-1,1) \sim$ $L$. SO $(p-1,1)$, where $L$ is not a group but a $K$-loop, a non-associative generalization of Abelian groups [12]. In this case, the deformation of the translation symmetry will be related to the $\mathrm{K}$ loop structure. The goal of the paper is to construct the quantum group $\mathcal{S}=\mathcal{C}(L) \rtimes k \mathrm{SO}(p-$ $1,1)$ - where the group algebra $k \mathrm{SO}(p-1,1)$ acts on the algebra of functions $\mathcal{C}(L)$ on the K-loop with the relevant Hopf structures. This quantum group can be seen once again as a deformation of the Poincaré Hopf algebra $\mathcal{C}\left(\mathbb{R}^{p}\right) \rtimes k \mathrm{SO}(p-1,1) \stackrel{\kappa}{\longrightarrow} \mathcal{C}(L) \rtimes k \mathrm{SO}(p-1,1)$, but not in the Hopf or quasi-Hopf algebra setting, since the antipode of $\mathcal{S}$ will not be antimultiplicative. This means in particular that this deformation has not been identified in Zakrzewski's classification of Poincaré deformations [6]. We will show that this quantum group $\mathcal{S}$ encodes the deformed Poincaré symmetry identified in [3, 4]. The infinitesimal structure of a K-loop is a Lie triple system and we will explain how Snyder non-commutative structure is naturally encoded in such structure. Lie triple systems provide therefore a new type of non-commutative spaces. This is the other main result of this paper. As a side result, one recovers that the $*$-product defined in $[3,4]$ is actually a realization of the Lie triple structure.

In the first section we recall the different notions of loop which can be found in the standard literature, emphasising on the K-loop case. In the second section we introduce the main result of the paper that is the construction of the quantum group encoding the symmetries of Snyder spacetime. In the third section, we show how to construct a scalar field action invariant under such symmetry, using the method described in [13]. In the fourth section, we recall the infinitesimal notion of a smooth K-loop given by Lie triple system and explain how this structure can be interpreted as encoding Snyder non-commutative geometry. The concluding section sets a list of interesting points to explore.

\section{K-loop: a review}

We recall in this section the basic definitions of the most studied loops with particular emphasis on the notion of K-loop, a (midely) non-associative generalization of Abelian group. Most of the content of this section (except Proposition 2, Definition 4 and Example 2) can be found in the standard reference on K-loops [12] (see also [14] for further references on the notion of loops).

Definition 1 (quasi-group and loop). A quasigroup $(S, \cdot)$ is a set $S$ with a binary operation "." such that for each $a$ and $b$ in $S$, there exist unique elements $x$ and $y$ in $S$ such that $a \cdot x=b, y \cdot a=b$. A quasigroup is a loop $L$ if it has also an identity $e$ such that $e \cdot a=a \cdot e=a$.

Note that this implies in particular that in a loop, we have a unique identity element and that the left and right inverse are unique.

Definition 2 (left Bol loop). Let $L$ be a loop. The left Bol identity is

$$
a \cdot(b \cdot(a \cdot c))=(a \cdot(b \cdot a)) \cdot c, \quad \forall a, b, c \in L .
$$

The right Bol identity is

$$
(c \cdot a) \cdot b) \cdot a=c \cdot((a \cdot b) \cdot a)), \quad \forall a, b, c \in L .
$$

A loop $L$ is said to be a left Bol loop (resp. right Bol loop) if it satisfies the left Bol identity (resp. the right Bol identity).

Proposition 1 (alternative loop). Let $L$ be a left Bol loop, then it is left alternative, that is

$$
a \cdot(a \cdot b)=a^{2} \cdot b=(a \cdot a) \cdot b .
$$


This proposition follows from the left Bol property with $b=1$. The consistency relationships between the product and inverse map of a loop will characterize different types of loop.

Definition 3 (inverse properties). Let $L$ be a loop.

- The automorphic inverse property (AIP) is $(a \cdot b)^{-1}=a^{-1} \cdot b^{-1}, \forall a, b \in L$.

- The inverse property (IP) is $(a \cdot b)^{-1}=b^{-1} \cdot a^{-1}, \forall a, b \in L$.

- The left inverse property (LIP) is $\exists a^{-1} \in L, a^{-1} \cdot(a \cdot b)=b, \forall a, b \in L$.

- The right inverse property (RIP) is $\exists b^{-1} \in L,(a \cdot b) \cdot b^{-1}=a, \forall a, b \in L$.

Note that the LIP together with the RIP gives the IP. Let us give now some examples of loops.

Definition 4 (Lie group). A group is a loop satisfying the inverse property and

$$
a \cdot(b \cdot c)=(a \cdot b) \cdot c=a \cdot b \cdot c .
$$

A group is therefore in particular a left and right Bol loop.

Definition 5 (Moufang loop). A Moufang loop is a left and right Bol loop. It satisfies in particular the inverse property.

Definition 6 (left K-loop). A left $K$ - loop (or left Bruck loop) is a left Bol loop satisfying the automorphic inverse property.

In [15] the authors focused on the Hopf structures related to the Moufang case, which are a natural non-associative generalization of non-Abelian groups. We are interested instead in the K-loop case. In the following, we shall omit the term "left" since we shall always consider left K-loops, unless specified otherwise. To generate loops, one can consider the factorization of Lie groups.

Proposition 2. Consider the group $\mathrm{SO}(p, 1)$. The decomposition $\mathrm{SO}(p, 1) \sim L$. $\mathrm{SO}(p-1,1)$ provides a unique decomposition

$$
g=a h, \quad g \in \mathrm{SO}(p, 1), \quad h \in \mathrm{SO}(p-1,1), \quad a \in L .
$$

The product of $\mathrm{SO}(p, 1)$ induces a product "." in $L$

$$
a b=(a \cdot b) h_{a b}, \quad \forall a, b \in L, \quad h_{a b} \in \mathrm{SO}(p-1,1) .
$$

The groupoid L is a smooth K-loop with product “". Its inverse map coincides with the group inverse map.

The same proposition holds for the Cartan decomposition $\mathrm{SO}(p, 1) \sim L^{\prime}$. $\mathrm{SO}(p)$, and the decomposition $\mathrm{SO}(p) \sim L^{\prime \prime} . \mathrm{SO}(p-1)$, i.e. both $L^{\prime}$ and $L^{\prime \prime}$ are K-loops. The proof for these cases is given in [12]. This proposition and its variants for the other decompositions can also be proved using the notion of Lie triple systems [16].

There is a weak form of commutativity and associativity present in a K-loop. When constructed from a group factorization as in the above decompositions, it takes the shape

$$
\begin{aligned}
& a \cdot b=h_{a b}(b \cdot a) h_{a b}^{-1}, \\
& a \cdot(b \cdot c)=(a \cdot b) \cdot\left(h_{a b} c h_{a b}^{-1}\right) .
\end{aligned}
$$

Hence K-loops can be seen as non-associative generalization of Abelian groups. To conclude this section, let us give two examples of K-loops. 
Example 1. Consider the orthochronous Lorentz group $\mathrm{SO}^{+}(3,1)$ and its Cartan decomposition $\mathrm{SO}^{+}(3,1) \sim L^{\prime}$. $\mathrm{SO}(3) . L^{\prime}$ is a K-loop $[17,18]$ which can be identified to the space of $3 \mathrm{D}$ speeds in Special Relativity, and the loop product encodes the speed addition used when changing reference frame. Let us recall its structure. $L^{\prime}$ is isomorphic to the $3 \mathrm{~d}$ upper hyperboloid $H_{3}^{+}$. This space can be embedded in $R^{4}$ as $H_{3}^{+}=\left\{V_{\mu} \in \mathbb{R}^{4}, V^{\mu} V_{\mu}=1, V_{0}>0\right\}$ and is generated by the boost $a=e^{i \frac{\eta}{2} \vec{b} \cdot \vec{K}}$, where $K_{i} \in \mathfrak{s o}(3,1)$ are the boost generators, $\eta$ is the boost angle, $\vec{b}$ is the boost vector $\left(\vec{b}^{2}=1\right)$, and we are using the spinorial representation of $\operatorname{SL}(2, \mathbb{C})[19,4]$. The product of boosts gives the loop product:

$$
\begin{gathered}
e^{i \frac{\eta_{1}}{2} \vec{b}_{1} \cdot \vec{K}} e^{i \frac{\eta_{2}}{2} \vec{b}_{2} \cdot \vec{K}}=e^{i \frac{\eta_{12}}{2} \vec{b}_{12} \cdot \vec{K}} R_{\eta_{i}, \vec{b}_{i}} \Longleftrightarrow a_{1} a_{2}=a_{12} h_{12} \\
\downarrow \\
e^{i \frac{\eta_{1}}{2} \vec{b}_{1} \cdot \vec{K}} \cdot e^{i \frac{\eta_{2}}{2} \vec{b}_{2} \cdot \vec{K}} \equiv e^{i \frac{\eta_{12}}{2} \vec{b}_{12} \cdot \vec{K}} \Longleftrightarrow a_{1} \cdot a_{2}=a_{12} .
\end{gathered}
$$

The rotation $R_{\eta_{i}, \vec{b}_{i}}$ is called the Thomas precession. The $3 \mathrm{~d}$ speed $\vec{v}$ in Special Relativity is given in terms of the coordinates on $\mathrm{H}_{3}^{+}$by

$$
\vec{v}(a) \equiv \vec{v}=c \tanh \eta \vec{b}=c \frac{\vec{V}}{V_{0}},
$$

where $c$ is the speed of light. The loop product (2.2) induces the sum of speeds:

$$
\begin{aligned}
\vec{v}\left(a_{1}\right) \oplus \vec{v}\left(a_{2}\right) & \equiv \vec{v}_{1} \oplus \vec{v}_{2}=c \tanh \eta_{12} \vec{b}_{12}=\vec{v}\left(a_{1} \cdot a_{2}\right) \\
& =\frac{1}{1+\frac{\vec{v}_{1} \cdot \vec{v}_{2}}{c^{2}}}\left(\left(1+\frac{\gamma_{1}}{1+\gamma_{1}} \frac{\vec{v}_{1} \cdot \vec{v}_{2}}{c^{2}}\right) \vec{v}_{1}+\frac{1}{\gamma_{1}} \vec{v}_{2}\right), \quad \gamma_{1}=\frac{1}{\sqrt{1-\frac{\vec{v}_{1}^{2}}{c^{2}}}} .
\end{aligned}
$$

It is not difficult to check that the inverse $\ominus$ coincides with the usual inverse $\ominus \vec{v}=-\vec{v}$ and this addition satisfies the automorphic inverse property $-\left(\vec{v}_{1} \oplus \vec{v}_{2}\right)=\left(-\vec{v}_{1}\right) \oplus\left(-\vec{v}_{2}\right)$. This K-loop can also be interpreted as momentum space in which case one reconstructs an Euclidian Snyder space-time $[3,4]$.

Example 2. Consider the decomposition $\mathrm{SO}(4,1) \sim L$. $\mathrm{SO}(3,1)$. In this case $L$ is isomorphic to the de Sitter space $d S$, which can be embedded in $\mathbb{R}^{5}$ as $d S=\left\{\pi_{A} \in \mathbb{R}^{5}, \pi^{A} \pi_{A}=-1\right\}$. It is generated by the de Sitter boosts $a=e^{i \frac{\eta}{2} B \cdot J_{4}}$, where $J_{4} \equiv J_{4 \mu} \in \mathfrak{s o}(4,1)(\mu=0, \ldots, 3)$, $\eta$ and $B^{\mu}$ are the angle and the boost vector $\left(B^{2}=-1\right)$ respectively and we are using the spinorial representation of $\mathrm{SO}(4,1)$. The de Sitter space is covered by two coordinate charts given by $\pi_{4}>0$ and $\pi_{4}<0$. As in the previous example, we restrict to the upper part of the de Sitter space, i.e. the sector with $\pi_{4}>0$ (which is stable under the Lorentz action). Snyder used the de Sitter space to define momentum space and momentum is given in terms of the coordinates on de Sitter space [1]

$$
p_{\mu}(a) \equiv p_{\mu}=\kappa \tanh \eta B_{\mu}=\kappa \frac{\pi_{\mu}}{\pi_{4}},
$$

$\kappa$ is the Planck mass ( $\hbar=c=1$ in this example). He did not define the notion of momenta addition, but we can use the K-loop structure to define it

$$
\begin{aligned}
e^{i \frac{\eta_{1}}{2} B_{1} \cdot J_{4}} e^{i \frac{\eta_{2}}{2} B_{2} \cdot J_{4}}=e^{i \frac{\eta_{12}}{2} B_{12} \cdot \Lambda_{\eta_{i}, B_{i}}} & \Longleftrightarrow a_{1} a_{2}=a_{12} h_{12} \\
& \downarrow \\
e^{i \frac{\eta_{1}}{2} B_{1} \cdot J_{4}} \cdot e^{i \frac{\eta_{2}}{2} B_{2} \cdot J_{4}} & \equiv e^{i \frac{\eta_{12}}{2} B_{12} \cdot J_{4}} \Longleftrightarrow a_{1} \cdot a_{2}=a_{12},
\end{aligned}
$$


where $\Lambda_{\eta_{i}, \vec{B}_{i}}$ is Lorentz transformation determined by the coordinates $\left(\eta_{i}, B_{i}\right)$. Using the expression (2.3), we obtain the addition of momenta $p_{\mu}\left(a_{1}\right) \oplus p_{\mu}\left(a_{2}\right) \equiv(p \oplus q)_{\mu}=\kappa \tanh \eta_{12} \vec{B}_{12}=$ $p_{\mu}\left(a_{1} \cdot a_{2}\right)$ given in the appendix. Since it is quite complicated we give here the first order contribution in $\kappa^{2}$ (with $\left.p \cdot q=p^{\mu} q_{\mu}, p^{2}=p^{\mu} p_{\mu}\right)$

$$
\begin{aligned}
& (p \oplus q)_{0}=p_{0}+q_{0}+\frac{1}{2 \kappa^{2}}\left(\left(p_{0}+2 q_{0}\right) p \cdot q+q_{0} p^{2}\right)+O\left(\frac{1}{\kappa^{3}}\right), \\
& (p \oplus q)_{i}=p_{i}+q_{i}+\frac{1}{2 \kappa^{2}}\left(\left(p_{i}+2 q_{i}\right) p \cdot q+\left(q_{i}-p_{i}\right) p_{0} q_{0}-p_{i} q_{0}^{2}+q_{i} \vec{p}^{2}\right)+O\left(\frac{1}{\kappa^{3}}\right) .
\end{aligned}
$$

Once again, it is easy to check that $-p$ is the inverse of the addition $\oplus$. A direct calculation shows that the addition $\oplus$ satisfies the automorphic inverse property law, that is $-\left(p_{1} \oplus p_{2}\right)=$ $\left(-p_{1}\right) \oplus\left(-p_{2}\right)$.

\section{Snyder quantum group}

In this section we present the main result of this paper, that is the construction of the quantum group encoding the symmetries of Snyder spacetime. Firstly, we define the notion of $K$-Hopf loop, which will be used to define the K-loop algebra $k L$, the analogue of the group algebra. Secondly, we define K-Hopf coloop which is the dual notion of K-Hopf loop. A specific example will be the algebra of functions on the K-loop $\mathcal{C}(L)$. Finally, we introduce the Snyder quantum group $\mathcal{C}(L) \rtimes k \mathrm{SO}(p-1,1)$ built out from cross product of the Lorentz group algebra with the algebra of functions on the K-loop.

\subsection{K-Hopf loop}

We define here the relevant Hopf structure for the K-loop. We use the Sweedler notation for the coproduct $\triangle a=a_{(1)} \otimes a_{(2)}$.

Definition 7 (K-Hopf loop). Let $k$ be a field of characteristic $\neq 2,3$ and $a, b \in \mathcal{H}$. A $K$-Hopf loop is a unital algebra $\mathcal{H}$, equipped with algebra homomorphisms $\triangle: \mathcal{H} \rightarrow \mathcal{H} \otimes \mathcal{H}, \epsilon: \mathcal{H} \rightarrow k$ forming a coassociative coalgebra, and a map $S: \mathcal{H} \rightarrow \mathcal{H}$ such that

$$
\begin{aligned}
& a_{(1)} \cdot\left(b \cdot\left(a_{(2)} \cdot c\right)\right)=\left(a_{(1)} \cdot\left(b \cdot a_{(2)}\right)\right) \cdot c, \\
& a_{(1)} \cdot\left(S\left(a_{(2)}\right) \cdot b\right)=b=S\left(a_{(1)}\right) \cdot\left(a_{(2)} \cdot b\right) .
\end{aligned}
$$

The equations (3.2) are the alter-ego of the left inverse property, whereas (3.1) is related to the left Bol property. The antipode $S$ is furthermore "multiplicative" and "comultiplicative"

$$
\begin{aligned}
& S(a \cdot b)=S(a) \cdot S(b), \\
& \triangle(S(a))=S\left(a_{(1)}\right) \otimes S\left(a_{(2)}\right) .
\end{aligned}
$$

This antipode is very different to the one met in a Hopf algebra [9] and in a Moufang Hopf quasigroup [15], since in these cases it is "antimultiplicative" and "anticomultiplicative".

Proposition 3. A K-Hopf loop satisfies

$$
\begin{aligned}
& a_{(1)} \cdot\left(a_{(2)} \cdot b\right)=\left(a_{(1)} \cdot a_{(2)}\right) \cdot b, \\
& S\left(a_{(1)}\right) \cdot a_{(2)}=a_{(1)} \cdot S\left(a_{(2)}\right) .
\end{aligned}
$$

Proof. (3.3) says that the K-Hopf loop is left alternative [15]. This is obtained from (3.1) by setting $b=1$. (3.4) is obtained from (3.2) applied on $a \otimes 1$. 
The group algebra $k G$ for a group $G$ provides a natural example of Hopf algebra [9]. In a similar way, the K-loop algebra $k L$ provides an example of K-Hopf loop.

Proposition 4. If $L$ is a $K$-loop, then $\mathcal{H}=k L$ is a $K$-Hopf loop with linear extension of the product and on the basis elements $a, b$ we have

$$
m(a \otimes b)=a \cdot b, \quad \epsilon(a)=1, \quad \triangle a=a \otimes a, \quad S(a)=a^{-1},
$$

and the unit is given by $e$.

The proof is straightforward. We notice in particular that $k L$ is both co-commutative and co-associative. The infinitesimal version of smooth K-loops is a Lie triple system [16]. The enveloping algebra for a Lie triple system has been constructed in [20]. This is another example of K-Hopf loop and can be seen as the infinitesimal version of $k L$, when $L$ is a smooth K-loop.

\section{$3.2 \quad$ K-Hopf coloop}

Once we have linearised the concept of K-loop into the K-loop algebra $k L$, one can reverse the arrows on all the maps, and obtain a K-Hopf coloop. We note $m$ the multiplication.

Definition 8 (K-Hopf coloop). Let $k$ be a field of characteristic $\neq 2,3$ and $f, f_{i} \in \mathcal{H}$. A $K$-Hopf coloop is a unital associative algebra $\mathcal{H}$, equipped with counital algebra homomorphisms $\triangle: \mathcal{H} \rightarrow \mathcal{H} \otimes \mathcal{H}, \epsilon: \mathcal{H} \rightarrow k$ and a linear map $\iota: \mathcal{H} \rightarrow \mathcal{H}$

$$
\begin{aligned}
& m\left(f_{(21)} \otimes f_{(1)}\right) \otimes f_{(221)} \otimes f_{(222)}=m\left(f_{(121)} \otimes f_{(11)}\right) \otimes f_{(122)} \otimes f_{(2)}, \\
& (m \otimes \mathrm{id})(\iota \otimes \mathrm{id} \otimes \mathrm{id})(\mathrm{id} \otimes \triangle) \triangle=1 \otimes \mathrm{id}=(m \otimes \mathrm{id})(\mathrm{id} \otimes \iota \otimes \mathrm{id})(\mathrm{id} \otimes \triangle) \triangle, \\
& \iota\left(m\left(f_{1} \otimes f_{2}\right)\right)=m\left(\iota f_{1} \otimes \iota f_{2}\right), \\
& \triangle(\iota f)=\iota f_{(1)} \otimes \iota f_{(2)} .
\end{aligned}
$$

By counital, we mean $(\mathrm{id} \otimes \epsilon) \triangle=(\epsilon \otimes \mathrm{id}) \triangle=\mathrm{id}$.

A K-Hopf coloop satisfies the analogue of Proposition 3, where the arrows are reversed.

Proposition 5. A K-Hopf coloop satisfies

$$
\begin{aligned}
& f_{(1)} \cdot f_{(21)} \otimes f_{(22)}=f_{(11)} \cdot f_{(12)} \otimes f_{2}, \\
& m(\iota \otimes i d) \triangle=\mathbb{I} \epsilon=m(i d \otimes \iota) \triangle .
\end{aligned}
$$

Proof. From (3.3), we have for $f \in k(L), a, b \in L$,

$$
\begin{aligned}
& \left(f, a_{(1)} \cdot\left(a_{(2)} \cdot b\right)\right)=\left(f,\left(a_{(1)} \cdot a_{(2)}\right) \cdot b\right) \\
& \quad \Leftrightarrow\left(f_{(1)} \cdot f_{(21)} \otimes f_{(22)}, a \otimes b\right)=\left(f_{(11)} \cdot f_{(12)} \otimes f_{2}, a \otimes b\right) .
\end{aligned}
$$

On the other hand, we have from (3.4)

$$
\left(f, a_{(1)} S a_{(2)}\right)=\left(f, S a_{(1)} a_{(2)}\right)=\epsilon(f) \epsilon(a) \Leftrightarrow(m(\iota \otimes \mathrm{id}) \triangle f, a)=(m(\mathrm{id} \otimes \iota) \triangle f, a) .
$$

As an example, we construct the K-Hopf coloop $k(L)$ - which is identified to the algebra of functions on $L$ - by duality, from the K-Hopf loop $k L$. We use the duality $(f, a) \equiv f(a)$, $\forall f \in k(L), a$ basis element in $k L$, and it is extended by linearity to all elements in $k L$.

Proposition 6. If $L$ is a K-loop, then $\mathcal{H}=k(L)$ is a K-Hopf coloop, with

$$
\begin{aligned}
& \left(m\left(f_{1} \otimes f_{2}\right), a\right)=f_{1}(a) f_{2}(a), \quad \epsilon(f)=f(e), \\
& (\triangle f, a \otimes b)=f(a \cdot b), \quad(\iota f, a)=f\left(a^{-1}\right),
\end{aligned}
$$

with $a, b$ elements in the basis of $k L$ and $f_{i} \in k(L)$, and the unit function is $\mathbb{I}$ such that $\mathbb{I}(a)=1$, $\forall a \in L$. 
The proof is straightforward. We notice that $k(L)$ is a commutative and associative algebra, but is not co-commutative nor co-associative.

The general Hopf coloop satisfies similar properties as in Proposition 3, which can be simply shown by reversing the arrows of the maps.

Example 3. Consider the K-Hopf loop generated from $L \sim \mathrm{SO}(4,1) / \mathrm{SO}(3,1)$ as given in Example 2. The dual structure is given by the set of functions $\mathcal{C}(L)$. The coordinate functions $p_{\mu} \in \mathcal{C}(L)$ is a natural example to consider. The coproduct $\triangle p_{\mu}$ is then the dual of the $\operatorname{sum}(2.4),(2.5)$

$$
\left\langle\left(\triangle p_{\mu}\right) ; a_{1} \otimes a_{2}\right\rangle=\left\langle p_{\mu} ; a_{1} \cdot a_{2}\right\rangle=p_{\mu}\left(a_{1} \cdot a_{2}\right)=(p \oplus q)_{\mu}, \quad a_{i} \in L .
$$

\subsection{Snyder Hopf loop}

We are now presenting the algebraic structure that is encoding the deformation of the Poincaré symmetry consistent with the Snyder space. We focus on the field $k=\mathbb{C}$. Considering the decomposition $\mathrm{SO}(p, 1) \sim L . \mathrm{SO}(p-1,1), L$ is isomorphic to the de Sitter space. There is a natural action of $\mathrm{SO}(p-1,1)$ on $L$ given in terms of the adjoint action $a \rightarrow u a u^{-1}=u \triangleright a$, $\forall a \in L, u \in \mathrm{SO}(p-1,1)$. This action is naturally lifted to the action of $k \mathrm{SO}(p-1,1)$ on $k(L)$, with $(u \triangleright f, a) \equiv f(u \triangleright a)$ and $u$ basis element of $k \mathrm{SO}(p-1,1)$. We recall that the group Hopf algebra $k \mathrm{SO}(p-1,1)$ is specified by the coproduct, antipode, counity and unity respectively defined on basis element $u \in \mathrm{SO}(p-1,1)$ (and extended by linearity),

$$
\triangle u=u \otimes u, \quad \mathbf{S} u=u^{-1}, \quad \epsilon(u)=1, \quad e .
$$

Proposition 7. $k(L)$ is a $k \mathrm{SO}(p-1,1)$-module algebra and coalgebra, i.e.

$$
\begin{aligned}
& u \triangleright\left(f_{1} \cdot f_{2}\right)=m\left(\triangle u \triangleright\left(f_{1} \otimes f_{2}\right)\right), \quad u \triangleright \mathbb{I}=\epsilon(u) \mathbb{I}=\mathbb{I}, \\
& \triangle(u \triangleright f)=\triangle u \triangleright \triangle f, \quad \epsilon(u \triangleright f)=\epsilon(u) \epsilon(f)=\epsilon(f),
\end{aligned}
$$

with $u$ basis elements of $k \mathrm{SO}(p-1,1)$ and we used (3.7). This is naturally extended by linearity. We have moreover

$$
\iota(u \triangleright f)=u \triangleright(\iota f) .
$$

Proof. We shall consider $a, b$ basis elements of $k L$ before extending naturally by linearity. First we prove (3.8),

$$
\begin{aligned}
\left(u \triangleright\left(f_{1} \cdot f_{2}\right), a\right) & =\left(\left(f_{1} \cdot f_{2}\right), u \triangleright a\right)=\left(f_{1} \otimes f_{2}, \triangle(u \triangleright a)\right)=\left(\left(f_{1} \otimes f_{2},(u \triangleright a) \otimes(u \triangleright a)\right)\right) \\
& \left.=\left(\left(f_{1} \otimes f_{2},(\triangle u) \triangleright(\triangle a)\right)\right)\right)=\left(\triangle u \triangleright\left(f_{1} \otimes f_{2}\right), \triangle a\right) \\
& =\left(m\left(\triangle u \triangleright\left(f_{1} \otimes f_{2}\right)\right), a\right) .
\end{aligned}
$$

We have moreover that

$$
(u \triangleright \mathbb{I}, a)=(\mathbb{I}, u \triangleright a)=\epsilon(u \triangleright a)=1=\epsilon(a)=(\mathbb{I}, a),
$$

where $\epsilon(u \triangleright a)$ is the counity on $k L$. Then we prove (3.9)

$$
\begin{aligned}
(\triangle(u \triangleright f), a \otimes b) & =(u \triangleright f, a \cdot b)=(f, u \triangleright(a \cdot b))=(f,(u \triangleright a) \cdot(u \triangleright b)) \\
& =(\triangle f,(u \triangleright a) \otimes(u \triangleright b))=((\triangle u) \triangleright(\triangle f), a \otimes b) .
\end{aligned}
$$

We have also

$$
\epsilon(u \triangleright f)=(u \triangleright f, e)=(f, e u \mathbf{S} u)=(f, e) \epsilon(u)=\epsilon(f) .
$$


Finally the proof of (3.10) goes as follows

$$
(\iota(u \triangleright f), a)=\left(u \triangleright f, a^{-1}\right)=\left(f, u \triangleright a^{-1}\right)=(\iota f, u \triangleright a)=(u \triangleright \iota f, a),
$$

where we used that the inverse of $L$ and $\mathrm{SO}(p-1,1)$ actually coincides with the inverse in $\mathrm{SO}(p, 1)$.

Since $k(L)$ is a $k \mathrm{SO}(p-1,1)$ module and co-module, we can consider the semi-direct product [9].

Definition 9 (Snyder Hopf loop). The Snyder Hopf loop $\mathcal{S} \equiv \mathcal{C}(L) \rtimes k \mathrm{SO}(p-1,1)$ is given in terms of the vector space $\mathcal{C}(L) \otimes k \mathrm{SO}(p-1,1)$, and respectively, product, coproduct, unit, counit, antipode and complex conjugate

Product $\quad\left(f_{1} \otimes u_{1}\right)\left(f_{2} \otimes u_{2}\right)=\left(f_{1} \cdot\left(u_{1} \triangleright f_{2}\right) \otimes u_{1} u_{2}\right)$,

Coproduct $\quad \triangle(f \otimes u)=\left(f_{(1)} \otimes u\right) \otimes\left(f_{(2)} \otimes u\right)$,

Unit and counit $\quad \mathbb{I} \otimes e, \quad \epsilon(f \otimes u)=f(e)$,

Antiopde $\quad \mathfrak{s}(f \otimes u)=\left(u^{-1} \triangleright \iota f \otimes u^{-1}\right)$,

Complex structure $(f \otimes u)^{*}=\left(\overline{u^{-1} \triangleright f} \otimes u^{-1}\right)$.

These relations are naturally extended by linearity when $u$ is a general element of $k \operatorname{SO}(p-1,1)$. The coproduct $\triangle f=f_{(1)} \otimes f_{(2)}$ is given in (3.6).

This construction can be extended to the other cases generated by the decompositions we mentioned above: $\mathcal{C}\left(L^{\prime \prime}\right) \rtimes k \mathrm{SO}(p-1)$ and $\mathcal{C}\left(L^{\prime}\right) \rtimes k \mathrm{SO}(p)$ are also Snyder Hopf loops. We have the following proposition

Proposition 8. The antipode in the Snyder Hopf loop is antimultitplicative but comultiplicative

$$
\begin{aligned}
& \left.\mathfrak{s}\left(\left(f_{1} \otimes u_{1}\right)\left(f_{2} \otimes u_{2}\right)\right)\right)=\mathfrak{s}\left(f_{2} \otimes u_{2}\right) \mathfrak{s}\left(f_{1} \otimes u_{1}\right), \\
& \triangle \mathfrak{s}=(\mathfrak{s} \otimes \mathfrak{s}) \triangle, \\
& m(\mathfrak{s} \otimes i d) \triangle=m(i d \otimes \mathfrak{s}) \triangle=\mathbb{I} \epsilon \otimes e \epsilon .
\end{aligned}
$$

\section{Proof.}

$$
\begin{aligned}
\left.\mathfrak{s}\left(\left(f_{1} \otimes u_{1}\right)\left(f_{2} \otimes u_{2}\right)\right)\right) & =\mathfrak{s}\left(f_{1} \cdot\left(u_{1} \triangleright f_{2}\right) \otimes u_{1} u_{2}\right)=\left(\left(u_{1} u_{2}\right)^{-1} \triangleright \iota\left(f_{1} \cdot\left(u_{1} \triangleright f_{2}\right)\right) \otimes\left(u_{1} u_{2}\right)^{-1}\right) \\
& =\left(\left(u_{1} u_{2}\right)^{-1} \triangleright\left(\iota f_{1} \cdot\left(u_{1} \triangleright \iota f_{2}\right)\right) \otimes\left(u_{1} u_{2}\right)^{-1}\right) \\
& =\left(\left(u_{1} u_{2}\right)^{-1} \triangleright\left(\iota f_{1}\right) \cdot\left(u_{2}^{-1} \triangleright\left(\iota f_{2}\right)\right) \otimes\left(u_{1} u_{2}\right)^{-1}\right),
\end{aligned}
$$

where we used Proposition 7, and the properties of the action

$$
\begin{aligned}
\mathfrak{s}\left(f_{2} \otimes u_{2}\right) \mathfrak{s}\left(f_{1} \otimes u_{1}\right) & =\left(u_{2}^{-1} \triangleright\left(\iota f_{2}\right) \otimes u_{2}^{-1}\right)\left(u_{1}^{-1} \triangleright\left(\iota f_{1}\right) \otimes u_{1}^{-1}\right) \\
& =\left(u_{2}^{-1} \triangleright\left(\iota f_{2}\right) \cdot\left(u_{2}^{-1} u_{1}^{-1}\right) \triangleright\left(\iota f_{1}\right) \otimes u_{2}^{-1} u_{1}^{-1}\right) .
\end{aligned}
$$

This is equal to (3.12) if we recall that the algebra $k(L)$ is commutative. Finally we show that the the antipode is comultiplicative

$$
\begin{aligned}
\triangle \mathfrak{s}(f \otimes u) & =\triangle\left(u^{-1} \triangleright \iota f \otimes u^{-1}\right)=\left(\left(\triangle u^{-1}\right) \triangleright(\triangle \iota f) \otimes \triangle u\right) \\
& =\left(\left(\triangle u^{-1}\right) \triangleright((\iota \otimes \iota) \triangle f) \otimes \triangle u\right)=(\mathfrak{s} \otimes \mathfrak{s}) \triangle(f \otimes u),
\end{aligned}
$$

where we used the consistency relations between the coproduct and antipode for $k(L)$ and $k \mathrm{SO}(p, 1)$ inherited from Proposition 7. The proof of (3.11) follows directly from Proposition 5 . 


\section{Scalar field theory}

Once we have defined the fundamental algebraic structure, we can proceed to construct a scalar field theory, invariant under such symmetry group, following the same route as [13]. We focus here on the $4 \mathrm{~d}$ Lorentzian case, i.e. $L \sim d S$ and we are going to show how to recover the results of $[3,4]$.

As in Example 2, we focus on the upper part of the de Sitter space $L^{+}$. We consider therefore the Snyder Hopf loop $\mathcal{S}^{+}=\mathcal{C}\left(L^{+}\right) \rtimes k \mathrm{SO}(3,1)$ which is naturally obtained from $\mathcal{S}$ by restricting the set of functions $\mathcal{C}(L)$ to $\mathcal{C}\left(L^{+}\right)$. This restriction is consistent with the Hopf structures given in Definition 9.

The scalar field is an element of the dual $\mathcal{D}^{*}$ of the coset $k\left(L^{+}\right) \sim \mathcal{S}^{+} / k \mathrm{SO}(3,1)$, which can be specified as the set of distributions with compact support $k^{*}\left(L^{+}\right) . \forall f \otimes u \in \mathcal{S}, v \in \mathrm{SO}(3,1)$

$$
\begin{aligned}
\mathcal{D}^{*}= & \left\{F \in \mathcal{S}^{+*} \mid\langle(f \otimes u)(\mathbb{I} \otimes v), F\rangle=\langle f \otimes u, F\rangle\right\} \\
& \sim k^{*}\left(L^{+}\right)=\left\{\Phi \mid\langle f, \Phi\rangle=\langle f \otimes e, F\rangle, f \in k\left(L^{+}\right)\right\} .
\end{aligned}
$$

This set of distributions is equipped with the convolution product which makes $k^{*}\left(L^{+}\right)$an algebra. The convolution product is constructed using the coproduct on $\mathcal{S}^{+}$,

$$
\left\langle f, \Phi_{1} \circ \Phi_{2}\right\rangle \equiv\left\langle\triangle_{\mathcal{S}}(f \otimes e), F_{1} \otimes F_{2}\right\rangle=\left\langle\triangle_{k(L)} f, \Phi_{1} \otimes \Phi_{2}\right\rangle .
$$

The scalar field can be seen as a function on $L^{+}$, which is then interpreted as momentum space. Hence, it is convenient to consider the subalgebra of distributions given by the algebra of functions (with compact support) $k\left(L^{+}\right) \subset \mathcal{D}^{*}$. For $\phi_{i} \in k\left(L^{+}\right)$, we have

$$
\phi_{1} \circ \phi_{2}(a)=\int\left[d a_{i}\right]^{2} \phi_{1}\left(a_{1}\right) \phi_{2}\left(a_{2}\right) \delta\left(a^{-1} \cdot\left(a_{1} \cdot a_{2}\right)\right) \text {, }
$$

where we are using the measure $[d a]=d^{5} \pi \delta\left(\pi_{A} \pi^{A}+1\right) \theta\left(\pi_{4}\right)$ on $L^{+}$, induced from the group decomposition and the loop product. This convolution product between functions is used to generate the relevant terms in the scalar field action. The propagating term and the $\phi^{3}$-like interaction term are given as

$$
\begin{aligned}
& \Psi \circ \phi(e)=\int[d a]^{2}\left(p^{2}\left(a_{1}\right)+m^{2}\right) \phi\left(a_{1}\right) \phi\left(a_{2}\right) \delta\left(a_{1} \cdot a_{2}\right), \quad \Psi(a)=\left(p^{2}(a)+m^{2}\right) \phi(a), \\
& \phi \circ(\phi \circ \phi)(e)=\int[d a]^{3} \phi_{1}\left(a_{1}\right) \phi_{2}\left(a_{2}\right) \phi_{2}\left(a_{2}\right) \delta\left(a_{1} \cdot\left(a_{2} \cdot a_{3}\right)\right),
\end{aligned}
$$

where the delta function encodes the generalization of the momentum conservation law and $p_{\mu}(a)$ is the Snyder coordinates associated to the loop element $a$. Note that the convolution product is not associative, since it is based on the loop product. It is therefore important to keep track of the order of the brackets. The action $\Sigma(\phi)$ for the scalar field $\phi$ reads explicitly

$$
\begin{aligned}
\Sigma(\phi)= & \Psi \circ \phi(e)+\frac{\lambda}{3 !} \phi \circ(\phi \circ \phi)(e)=\int[d p]^{2} \phi\left(p_{1}\right)\left(p_{1}^{2}+m^{2}\right) \phi\left(p_{2}\right) \delta\left(p_{1} \oplus p_{2}\right) \\
& +\frac{\lambda}{3 !} \int[d p]^{3} \phi\left(p_{1}\right) \phi\left(p_{2}\right) \phi\left(p_{3}\right) \delta\left(p_{1} \oplus\left(p_{2} \oplus p_{3}\right)\right) .
\end{aligned}
$$

In the second line, we have expressed the action in terms of the Snyder coordinates, in particular the measures reads $\kappa^{4}[d a]=[d p]=\frac{1}{2} d^{4} p\left(\left(1-\frac{p^{2}}{\kappa^{2}}\right)\right)^{-\frac{5}{2}}$. The sum of momenta is given by $(2.4),(2.5)$.

We need to check that the Snyder Hopf loop $\mathcal{S}^{+}$encodes the right symmetry for this scalar action. We need therefore to define how the field $\phi$ is transforming under the quantum group action. The symmetry action of $\mathcal{S}^{+}$on $k^{*}\left(L^{+}\right)$is induced by its action on $\mathcal{S}^{*}$

$$
\langle f,(a \otimes u) \triangleright \phi\rangle \equiv\langle f \otimes e,(a \otimes u) \triangleright F\rangle=\left\langle(a \otimes u)^{*}(f \otimes e), F\right\rangle .
$$


This means concretely that the Lorentz group $\mathrm{SO}(3,1)$ sector acts by the adjoint action, whereas the $k\left(L^{+}\right)$sector acts by multiplication. This last sector encodes the deformed translation symmetry

$$
\phi(a) \rightarrow \phi(u \triangleright a), \quad \phi(a) \rightarrow f(a) \phi(a) .
$$

To determine the exact realization of the translation symmetry, one needs to introduce spacetime, through a Fourier transform. We consider therefore the c-numbers $x_{\mu} \in \mathbb{R}^{4}$ and the plane-wave $e^{i p \cdot x}$. To keep track of the modified sum of momenta (2.4), (2.5), we introduce the modified product $*$ between plane-waves

$$
e^{i p_{1} \cdot x} * e^{i p_{2} \cdot x} \equiv e^{i\left(p_{1} \oplus p_{2}\right) \cdot x} .
$$

We can then introduce the Fourier transform of the field $\phi \in \mathcal{C}\left(L^{+}\right)$

$$
\hat{\phi}(x) \equiv \int[d a] e^{i p(a) \cdot x} \phi(a)=\int[d p] e^{i p \cdot x} \phi(p) .
$$

The product between fields is given by the $*$-product, which is the dual of the convolution product

$$
\begin{aligned}
\left(\hat{\phi}_{1} * \hat{\phi}_{2}\right)(x) & =\int[d p]^{2} e^{i p_{1} \cdot x} * e^{i p_{2} \cdot x} \phi_{1}\left(p_{1}\right) \phi_{2}\left(p_{2}\right) \\
& =\int[d p]^{3} e^{i p \cdot x} \phi_{1}\left(p_{1}\right) \phi_{2}\left(p_{2}\right) \delta\left(-p \oplus\left(p_{1} \oplus p_{2}\right)\right)=\int[d p] e^{i p \cdot x} \phi_{1} \circ \phi_{2}(p) .
\end{aligned}
$$

With this Fourier transform, the scalar field action becomes

$$
\Sigma(\phi)=\int[d x]\left(-\left(\partial^{\mu} \hat{\phi}\right) *\left(\partial_{\mu} \hat{\phi}\right)+m^{2} \hat{\phi} * \hat{\phi}+\frac{\lambda}{3 !} \hat{\phi} *(\hat{\phi} * \hat{\phi})\right) .
$$

The translation action is now given as $x \rightarrow x+\varepsilon$ and the field $\phi(p)$ is transforming therefore as $\phi(p) \rightarrow e^{i p \cdot \varepsilon} \phi(p)$, which gives us therefore $f(p)=e^{i p \cdot \varepsilon}$ for the symmetry action (4.3).

To prove invariance of the action under $\mathcal{S}^{+}$, we also need to know how it is acting on convolution product. This is constructed naturally using the coproduct of $\mathcal{S}^{+}$and the coproduct on $k\left(L^{+}\right)$as in $(3.6)$

$$
(f \otimes u) \triangleright\left(\phi_{1} \circ \phi_{2}\right) \equiv\left(\left(f_{(1)} \otimes u\right) \triangleright \phi_{1}\right) \circ\left(\left(f_{(1)} \otimes u\right) \triangleright \phi_{2}\right) .
$$

It is straightforward to check that the action $\Sigma(\phi)$ constructed from the terms (4.1) and (4.2) is then invariant under such transformations. The coproduct of $\mathcal{S}^{+}$also provides the action of the Lorentz group and the translations on a tensor product of fields

$$
\phi\left(p_{1}\right) \phi\left(p_{2}\right) \rightarrow \phi\left(u \triangleright p_{1}\right) \phi\left(u \triangleright p_{2}\right), \quad \phi\left(p_{1}\right) \phi\left(p_{2}\right) \rightarrow e^{i\left(p_{1} \oplus p_{2}\right) \cdot \varepsilon} \phi\left(p_{1}\right) \phi\left(p_{2}\right) .
$$

The deformation of the translation symmetry is therefore naturally encoded in the loop product.

Before closing this section, we can evaluate explicitely the star product between different typical functions, using the properties of the Fourier transform we have introduced. For example, we can calculate the commutator of coordinates with the $*$-product $[3,4]$

$$
\left[x_{\mu}, x_{\nu}\right]_{*}=0 .
$$

The commutator of monomes of the first degree is zero. This is quite different than the usual noncommutative spaces. However, this does not mean that we have a commutative space. Indeed, for example the commutator of a monome of the second degree with one of the first degree 
is not zero due to the non-associativity. As an example, let us look at the more complicated combination:

$$
\left\langle x_{\mu}, x_{\nu}, x_{\alpha}\right\rangle \equiv x_{\mu} *\left(x_{\nu} * x_{\alpha}\right)-x_{\nu} *\left(x_{\mu} * x_{\alpha}\right)=-\frac{1}{\kappa^{2}}\left(\eta_{\nu \alpha} x_{\mu}-\eta_{\mu \alpha} x_{\nu}\right)=\frac{i}{\kappa^{2}} J_{\mu \nu} \triangleright x_{\alpha},
$$

where $J_{\mu \nu} \in \mathfrak{s o}(3,1)$ acts in the usual way on the coordinates $x_{\alpha}$. By introducing a Weyl map, we are going to show in the next section how this star product is the realization of the noncommutative structure implemented by a Lie triple system, the infinitesimal version of a smooth K-loop.

\section{$5 \quad$ Snyder space as a Lie triple system}

In the first subsection, we first recall the different examples of Bol algebras which are the infinitesimal version of the different smooth Bol loops we considered in Section 2. We focus on the Lie triple system case and show how given a Lie triple system, we can recover Snyder commutation relations, through Jacobson's embedding theorem. In the second subsection, we define the Weyl map and recover the star product we have introduced.

\subsection{Lie triple system}

In Section 2, we have first introduced the concept of Bol loop. The concept of Bol algebra is the infinitesimal version of a smooth Bol loop.

Definition 10 (left Bol algebra, see [20] and references therein). A left Bol algebra is a vector space $\mathcal{B}$ equipped with a bilinear and a trilinear product, noted respectively $[,]_{B}$ and $\langle,$,$\rangle , satisfying the following properties X, Y, Z, U, V \in \mathcal{B}$

$$
\begin{aligned}
& \langle X, Y, Z\rangle=-\langle Y, X, Z\rangle, \quad[X, Y]_{B}=-[Y, X]_{B} \\
& \langle X, Y, Z\rangle+\langle Y, Z, X\rangle+\langle Z, X, Y\rangle=0 \\
& \langle U, V,\langle Z, X, Y\rangle\rangle=\langle\langle U, V, Z\rangle, X, Y\rangle+\langle Z,\langle U, V, X\rangle, Y\rangle+\langle Z, X,\langle U, V, Y\rangle\rangle \\
& \left\langle U, V,[X, Y]_{B}\right\rangle=[\langle U, V, X\rangle, Y]_{B}+[X,\langle U, V, Y\rangle]_{B}+[X,\langle U, V, Y\rangle]_{B} \\
& +\langle X, Y,[U, V]\rangle+[[U, V],[X, Y]]_{B} .
\end{aligned}
$$

(5.1) encodes the Jacobi identity for the trilinear operation. (5.2) means that the map $\delta_{X, Y}: \mathcal{B} \rightarrow \mathcal{B}$ defined as $\delta_{X, Y}(Z)=\langle X, Y, Z\rangle$ is a derivation for the ternary operation. Explicitly, if $D$ is a derivation $D$ for the trilinear product one has

$$
D\langle X, Y, Z\rangle=\langle D X, Y, Z\rangle+\langle X, D Y, Z\rangle+\langle X, Y, D Z\rangle .
$$

Proposition 9. Given a Bol loop L, the bilinear and trilinear products $[,]_{B},\langle,$,$\rangle of the Bol$ algebra are obtained as follows [21]:

$$
\begin{aligned}
& \left.\qquad X_{1}, X_{2}\right]_{B} \equiv-\frac{1}{2} \frac{d^{2}}{d t_{1} d t_{2}}\left(\left(a_{1} \cdot a_{2}\right) \cdot\left(a_{2} \cdot a_{1}\right)^{-1}\right), \\
& \left(X_{1}, X_{2}, X_{3}\right) \equiv i \frac{1}{3 !} \frac{d^{3}}{d t_{1} d t_{2} d t_{3}}\left(\left(\left(a_{1} \cdot a_{2}\right) \cdot a_{3}\right) \cdot\left(a_{1} \cdot\left(a_{2} \cdot a_{3}\right)\right)^{-1}\right), \\
& \left\langle X_{1}, X_{2}, X_{3}\right\rangle=2\left(X_{1}, X_{3}, X_{2}\right)+\left[[X, Y]_{B}, Z\right]_{B}, \\
& \text { with } X_{i}=\left.\frac{d}{d t_{i}} a_{i}\right|_{t_{i}=0}=\left.\frac{d}{d t_{i}} e^{i t_{i} X_{i}}\right|_{t_{i}=0} .
\end{aligned}
$$

We can identify now the infinitesimal structures behind the most well known examples of Bol loops. 
Example 4. A Lie algebra $\mathfrak{g}$ with product [, ] is a Bol algebra such that

$$
[X, Y]_{B} \equiv[X, Y], \quad\langle X, Y, Z\rangle \equiv 0 .
$$

This can be derived from Proposition 9 using the loop given by a Lie group. We recover that a Lie algebra is the infinitesimal version of a Lie group.

Definition 11 (Mal'tsev algebra, see [20] and references therein). A Mal'tsev algebra is a left Bol algebra such that

$$
\langle X, Y, Z\rangle=\left[[x, y]_{B}, z\right]_{B}-\frac{1}{3} J(x, y, z), \quad[J(x, y, z), x]_{B}=J\left(x, y,[x, z]_{B}\right)
$$

with

$$
J(x, y, z)=\left[[x, y]_{B}, z\right]_{B}+\left[[z, u]_{B}, y\right]_{B}+\left[[y, z]_{B}, x\right]_{B} .
$$

Such algebra can be seen as the infinitesimal version of a smooth Moufang loop as it can be checked using Proposition 9.

We introduce now the relevant example for studying Snyder space-time.

Definition 12 (Lie triple system, see [20, 22, 16] and references therein). A Lie triple system $\ell$ is a left Bol algebra such that

$$
[X, Y]_{B}=0, \quad \forall x, y \in \ell
$$

It is therefore totally determined in terms of the trilinear product which has the following properties

$$
\begin{aligned}
& \langle X, Y, Z\rangle=-\langle Y, X, Z\rangle \\
& \langle X, Y, Z\rangle+\langle Y, Z, X\rangle+\langle Z, X, Y\rangle=0, \\
& \langle U, V,\langle Z, X, Y\rangle\rangle=\langle\langle U, V, Z\rangle, X, Y\rangle+\langle Z,\langle U, V, X\rangle, Y\rangle+\langle Z, X,\langle U, V, Y\rangle\rangle .
\end{aligned}
$$

In fact a direct calculation from (5.3) shows that we have

$$
\langle X, Y, Z\rangle=2(Z, X, Y)=[[X, Y], Z]=(X Y-Y X) Z-Z(X Y-Y X) .
$$

This structure can be seen as the infinitesimal version of a smooth K-loop [16], once again using Proposition 9.

Lie triple systems have been studied by mathematicians and most of the results found in the context of Lie algebras have been analysed in the Lie triple system context, see [16, 23, 24] and references therein.

The property (5.5) indicates that the trilinear product can be expressed in terms of some Lie algebra bracket. The following theorem makes this statement more precise.

Theorem 1 (standard imbedding [22, 24]). Let $\ell$ a Lie triple system, and for $X, Y \in \ell$, we note $\delta_{X, Y}(Z)$ the linear transformation $Z \rightarrow\langle X, Y, Z\rangle$ and $\mathfrak{m}$ the set of all such linear transformations. Then $\mathfrak{m}$ is a Lie algebra and moreover the vector space $\mathcal{G} \equiv \ell \oplus \mathfrak{m}$ is a Lie algebra with bracket

$$
\begin{aligned}
& {[X, Y]=\delta_{X, Y}, \quad[A, B]=A B-B A,} \\
& {[A, X]=-[X, A]=A X, \quad \forall A, B \in \mathfrak{m}, \quad \forall X, Y \in \mathfrak{p} .}
\end{aligned}
$$

The map $\sigma: \mathcal{G} \rightarrow \mathcal{G}$, such that $\sigma(X)=-X, \sigma(A)=A$ is an involutive automorphism of $\mathcal{G}$. 
Notice that the bracket appearing in this construction has nothing to do with the Bol bracket $[,]_{B}$.

Remark 1. From the property (5.2), we notice that $\delta_{X, Y}$ is a derivation. One can restate the previous theorem by saying that one can make $\operatorname{Der}_{\ell} \oplus \ell$ a Lie algebra, where $\operatorname{Der}_{\ell}$ is the set of (inner) derivations of $\ell$.

Proof. To prove the Theorem 1, one has to check that the Lie bracket satisfies the Jacobi identity. Following the property (5.4), it is clear that we have for example

$$
[[X, Y], Z]+[[Z, X], Y]+[[Y, Z], X]=0 .
$$

The relation

$$
[[A, Y], Z]+[[Z, A], Y]+[[Y, Z], A]=0
$$

states that $A=\delta_{X, Y}$ is a derivation. This relation is shown by straightforward calculations. The other relations follow also by direct calculations.

Conversely, given a Lie algebra $\mathcal{G}$ and an involutive automorphism $\sigma$, one can easily construct a Lie triple system. Indeed, since $\sigma$ is an involution, it has two eigenvalues -1 , 1 , with respective eigenspaces noted $\ell, \mathfrak{m}$, such that $\mathcal{G}=\ell \oplus \mathfrak{m}$. $\sigma$ is an automorphism, therefore using the compatibility with the product, it is easy to check that $[\ell, \ell] \subset \mathfrak{m},[\ell, \mathfrak{m}] \subset \ell$ and $[\mathfrak{m}, \mathfrak{m}] \subset \mathfrak{m}$. The vector space $\ell$ equipped with the trilinear product $\langle X, Y, Z\rangle=[[X, Y], Z]$ constructed from the Lie algebra bracket $\langle,$,$\rangle is a Lie triple system.$

This theorem and its converse are the key to understand the nature of Snyder spacetime. The following example shows how to construct a Lie triple system from $\mathfrak{s o}(p, 1)$ and recover Snyder non-commutative structure.

Example 5. The decomposition of the Lie algebra $\mathfrak{s o}(p, 1) \sim \ell \oplus \mathfrak{s o}(p-1,1)$ is given by the involutive automorphism $\sigma\left(J_{p \mu}\right)=-J_{p \mu}$ and $\sigma\left(J_{\mu \nu}\right)=J_{\mu \nu}$ with $\mu=0, \ldots, p-1$ and $J_{\mu \nu} \in \mathfrak{s o}(p-1,1)$. The sector $\ell$ is generated by the "de Sitter boosts" $J_{p \mu}$ and is therefore a Lie triple system. We have following Jacobson's theorem

$$
\left\langle J_{p \mu}, J_{p \nu}, J_{p \alpha}\right\rangle=\eta_{\nu \alpha} J_{p \mu}-\eta_{\mu \alpha} J_{p \nu}
$$

Conversely, given the Lie triple system $\ell$, we recover the Snyder commutation relation by introducing the dimensionful coordinates

$$
X_{\mu}=\frac{1}{\kappa} J_{p \mu},
$$

which satisfy therefore, still thanks to Jacobson's theorem

$$
\left[X_{\mu}, X_{\nu}\right] \equiv \delta_{X_{\mu}, X_{\nu}}=\left\langle X_{\mu}, X_{\nu}, .\right\rangle=\frac{1}{\kappa^{2}} J_{\mu \nu}
$$

This can be naturally extended to the case $\mathfrak{s o}(p, q)$. Coming back to the example we have considered in Section 4, the smooth K-loop $L \sim \mathrm{SO}(4,1) / \mathrm{SO}(3,1)$ is associated with the Lie triple system of Example 5. The operator $X_{\mu} \sim \frac{1}{\kappa} J_{p \mu}$ can be viewed as a distribution (with support the identity element) as follows. Given a test function $f \in \mathcal{C}\left(L^{+}\right)$,

$$
\left(X_{\mu}, f\right) \equiv-\left.2 \kappa i \frac{d}{d \eta} f\left(e^{i \frac{\eta}{2} J_{p \mu}}\right)\right|_{\eta=0} .
$$


The bilinear and trilinear products are then constructed in a similar way as in Proposition 9

$$
\begin{aligned}
& \left(\left[X_{1}, X_{2}\right]_{B}, f\right) \equiv i \frac{1}{2} \frac{d^{2}}{d t_{1} d t_{2}} f\left(\left(a_{1} \cdot a_{2}\right) \cdot\left(a_{2} \cdot a_{1}\right)^{-1}\right) \\
& \left(\left\langle X_{1}, X_{2}, X_{3}\right\rangle, f\right) i \equiv \frac{1}{3} \frac{d^{3}}{d t_{1} d t_{2} d t_{3}} f\left(\left(\left(a_{1} \cdot a_{2}\right) \cdot a_{3}\right) \cdot\left(a_{1} \cdot\left(a_{2} \cdot a_{3}\right)\right)^{-1}\right) .
\end{aligned}
$$

This means in particular that there is two types of bracket one should not confuse. On one hand there is the Bol bracket $\left[X_{\mu}, X_{\nu}\right]_{B}=0$ which is trivially zero since we are dealing with a K-loop (and especially the automorphic property). On the other hand, there is the bracket associated with the imbedding Lie algebra $\left[X_{\mu}, X_{\nu}\right] \equiv \delta_{X_{\mu}, X_{\nu}}$ from which the trilinear product is constructed as in (5.5). The Snyder commutation relations are encoded in this bracket and the natural algebraic framework to describe the Snyder space-time given by the Lie triple system structure.

\subsection{Weyl map}

We have seen in the last section that given a Lie triple system, Jacobson's theorem allows to recover the Snyder commutation relation. It is therefore natural to consider the set of functions on Snyder spacetime as the set of functions on the Lie triple system.

The notion of enveloping algebra $U(\mathcal{B})$ has been constructed for Bol algebras, and in particular it satisfies the Poincaré-Birkhoff-Witt theorem; we refer to [20] for the details. As a consequence, such enveloping algebra $U(\ell)$ exists for a Lie triple system $\ell$. This enveloping algebra $U(\ell)$ can be interpreted as the algebra of non-commutative functions $\mathcal{C}(\ell)$ on Snyder space-time, generated from the coordinates operators $X_{\mu}$.

We want to check now that the star product we have introduced in Section 4 is related to the non-commutative structure encoded in the Lie triple system. For this we need to introduce a Weyl map, an algebra isomorphism between the algebra of functions on the Lie triple system $\mathcal{C}(\ell)$ and the algebra of functions with the star product $\mathcal{C}_{*}\left(\mathbb{R}^{4}\right)$.

$$
\begin{aligned}
& \mathcal{W}: \mathcal{C}(\ell) \rightarrow \mathcal{C}_{*}\left(\mathbb{R}^{4}\right), \\
& \hat{f}(X) \equiv \int_{d S^{+}}[d p] f(p) e^{i p \cdot X} \rightarrow \mathcal{W}(\hat{f})(x) \equiv \int_{d S^{+}}[d p] f(p) e^{i p \cdot x},
\end{aligned}
$$

where $p$ is a momentum coordinate choice on the upper part of the de Sitter space, $[d p]$ the measure on the upper part of the de Sitter space, $e^{i p \cdot X} \in L$ and $f(p) \in \mathcal{C}(L)$. For example, for the generator $X_{\mu}$, we have $\mathcal{W}\left(X_{\mu}\right)(x) \equiv x_{\mu}$. The Weyl map is an isomorphism of algebra

therefore by definition we need to have $\mathcal{W}(\hat{f} \cdot \hat{h})(x) \equiv(\mathcal{W}(\hat{f}) * \mathcal{W}(\hat{h}))(x)$. The definition of the Weyl map (5.6) makes sure that the $*$-product we are using is precisely the one defined in Section 4. We have in particular

$$
\mathcal{W}\left(X_{\mu} X_{\nu}\right)(x)=x_{\mu} * x_{\nu}
$$

It is then immediate to check that

$$
\mathcal{W}\left(\left[X_{\mu}, X_{\nu}\right]_{B}\right)=0=\mathcal{W}\left(X_{\mu} X_{\nu}-X_{\nu} X_{\mu}\right)=x_{\mu} * x_{\nu}-x_{\nu} x_{\mu}=0,
$$

in accordance with (4.4). To consider the mapping of a product of three operators, we need to be careful since we have to take into account the non-associativity. Indeed, the position operator $X_{\mu}$ acts by (*-)multiplication of $x_{\mu}$

$$
X_{\mu} \triangleright \hat{f}(x) \equiv x_{\mu} * \hat{f}(x),
$$


and we use the natural action on the left, so that when considering the product of operators, we have

$$
\left.\left(X_{\mu} X_{\nu}\right) \triangleright \hat{f}(x)\right) \equiv X_{\mu} \triangleright\left(X_{\nu} \triangleright \hat{f}(x)\right)=x_{\mu} *\left(x_{\nu} * \hat{f}(x)\right) .
$$

This is important to keep in mind since we are using now a non-associative structure: order does matter. The product of operators is therefore naturally ordered from the right to the left. We have then

$$
\mathcal{W}\left(X_{\mu} X_{\nu} X_{\alpha}\right)=x_{\mu} *\left(x_{\nu} * x_{\alpha}\right) .
$$

When considering the triple product, the commutator does contribute in a non trivial way due to the non-associativity

$$
\begin{aligned}
\mathcal{W}\left(\left\langle X_{\mu}, X_{\nu}, X_{\alpha}\right\rangle\right) & =\mathcal{W}\left(\left[\left[X_{\mu}, X_{\nu}\right], X_{\alpha}\right]\right)=\mathcal{W}\left(\frac{i}{\kappa^{2}}\left[J_{\mu \nu}, X_{\alpha}\right]\right) \\
& =\mathcal{W}\left(\left(X_{\mu} X_{\nu}-X_{\nu} X_{\mu}\right) X_{\alpha}-X_{\alpha}\left(X_{\mu} X_{\nu}-X_{\nu} X_{\mu}\right)\right) \\
& \left.=x_{\mu} *\left(x_{\nu} * x_{\alpha}\right)-x_{\nu} *\left(x_{\mu} * x_{\alpha}\right)-x_{\alpha} *\left(x_{\mu} * x_{\nu}-x_{\nu} * x_{\mu}\right)\right) \\
& =\frac{i}{\kappa^{2}}\left[J_{\mu \nu}, x_{\alpha}\right]=\left\langle x_{\mu}, x_{\nu}, x_{\alpha}\right\rangle,
\end{aligned}
$$

where we have used (4.4), so that the last term in (5.7) is zero and we have used the result in (4.5). The Weyl map preserves, as required, the Lie triple system structure.

The Snyder commutation relation $\left[X_{\mu}, X_{\nu}\right]$ can also be obtained from the $*$-product using its action a function $\hat{f}(x)$, which we take to be $x_{\alpha}$ for simplicity $[3,4]$

$$
\left[X_{\mu}, X_{\nu}\right] \triangleright x_{\alpha}=\left(X_{\mu} X_{\nu}-X_{\mu} X_{\nu}\right) \triangleright x_{\alpha}=x_{\mu} *\left(x_{\nu} * x_{\alpha}\right)-x_{\nu} *\left(x_{\mu} * x_{\alpha}\right)=\frac{i}{\kappa^{2}} J_{\mu \nu} \triangleright x_{\alpha} .
$$

This shows that the commutator of the position operators does satisfy

$$
\left[X_{\mu}, X_{\nu}\right]=i \frac{1}{\kappa^{2}} J_{\mu \nu}
$$

\section{Concluding remarks}

There are two ways to interpret Snyder commutation relations. The first one is to say that this non-commutative space is actually a subspace of a bigger non-commutative space generated from the coordinates algebra $\left(X_{\alpha}, J_{\mu \nu}\right)$. The coordinates $J_{\mu \nu}$ are interpreted as coordinates describing extra dimensions. This is the perspective followed in the Doplicher-FredenhagenRoberts model [25] which can be seen then as an abelianization of the Snyder model [4, 26] with this interpretation. The second possibility is to consider space-time as only generated by the $X_{\mu}$. In this case, we have to face the issue that the coordinates commutations do not close. We argued in this paper that the solution of this issue is to consider Snyder space-time given in terms of a Lie triple system, that is instead of the bilinear product (5.8), one considers the trilinear product

$$
\left\langle X_{\mu}, X_{\nu}, X_{\alpha}\right\rangle \equiv\left[\left[X_{\mu}, X_{\nu}\right], X_{\alpha}\right]=\frac{1}{\kappa^{2}}\left(\eta_{\nu \alpha} X_{\mu}-\eta_{\mu \alpha} X_{\nu}\right) .
$$

In this case, one can construct a meaningful notion of non-commutative algebra of functions using the notion of enveloping algebra for Lie triple system which is dual, using a generalized Fourier transform, to the algebra of functions on the K-loop $L \sim \mathrm{SO}(p, 1) / \mathrm{SO}(p-1,1)$. On the 
other hand, given the Lie triple system, Jacobson's theorem tells us how to embedd it into a Lie algebra and to recover the Snyder commutation relations, from the trilinear product

$$
\left[X_{\mu}, X_{\nu}\right] \equiv \delta_{X_{\mu}, X_{\nu}}=\left\langle X_{\mu}, X_{\nu}, .\right\rangle=\frac{1}{\kappa^{2}} J_{\mu \nu}
$$

Lie triple systems provide therefore a new type of non-commutative geometry. This noncommutative geometry can be seen as flat since one can identify a quantum group, a deformation of the the Poincaré group, which acts on this space in a consistent way. We provided here the definition of this new quantum group, which we called Snyder quantum group, using the structure of K-loop. This latter can be be interpreted as momentum space as in Snyder's initial idea. The K-loop structure provides a rule to add momenta, an addition which is non-commutative and non-associative, just like the speed addition in Special Relativity. We have also presented how we can construct a scalar field action on a Lie triple system using a star product realization and how this action is invariant under the Snyder quantum group, encoding the analogue of the flat symmetries.

This work opens up a number of questions which are of interests for either mathematicians or theoretical physicists.

- The Snyder quantum group needs to be studied in details: the analysis of the representation theory should be performed. It would be interesting to check if the weak notion of commutativity (2.1) is related to a notion of braiding.

- The classification of the bi-covariant differential calculus [27] for Snyder space-time should be performed to make the analysis of the conserved currents [11].

- Now that we have a well defined classical action for a scalar field, it would be interesting to check if this non-commutative space fullfils Snyder's hope, that is whether a quantum field theory living in this space has no UV divergences.

\section{A Snyder sum}

In this appendix we present the calculations to determine the modified sum of momenta (2.4), (2.5) in Snyder's coordinates. We calculate the product of de Sitter boosts $a_{i}=\cosh \frac{\eta_{i}}{2} \mathrm{Id}+$ $2 i \sinh \frac{\eta_{i}}{2} B^{\mu} J_{4 \mu}$

$$
a_{1} a_{2}=a \Lambda R
$$

where $\Lambda=\cosh \frac{\alpha}{2} \operatorname{Id}+2 i \sinh \frac{\alpha}{2} b^{i} J_{0 i}$ is a boost and $R=\cos \frac{\theta}{2} \operatorname{Id}+i \sin \frac{\theta}{2} r^{i} J_{i}$ is a rotation. The term $a$ will encode the sum of momenta and $\Lambda R$ is the Lorentz precession, the generalization of the Thomas precession to the $4 \mathrm{~d}$ Lorentzian case [28]

$$
\begin{aligned}
& \cosh \frac{\eta_{1}}{2} \cosh \frac{\eta_{2}}{2}-\sinh \frac{\eta_{1}}{2} \sinh \frac{\eta_{2}}{2} B_{1} \cdot B_{2}=\cosh \frac{\eta}{2} \cosh \frac{\alpha}{2} \cos \frac{\theta}{2} \\
& \sinh \frac{\eta_{1}}{2} \sinh \frac{\eta_{2}}{2}\left(\vec{B}_{1} \wedge \vec{B}_{2}\right)^{i}=\cosh \frac{\eta}{2} \cosh \frac{\alpha}{2} \cos \frac{\theta}{2} r^{i} \\
& \sinh \frac{\eta_{1}}{2} \sinh \frac{\eta_{2}}{2}\left(B_{1}^{0} B_{2}^{i}-B_{1}^{i} B_{2}^{0}\right)=\cosh \frac{\eta}{2} \sinh \frac{\alpha}{2}\left(\cos \frac{\theta}{2} b^{i}+\sin \frac{\theta}{2}(\vec{b} \wedge \vec{r})^{i}\right) \\
& \cosh \frac{\eta_{1}}{2} \sinh \frac{\eta_{2}}{2} B_{2}^{0}+\cosh \frac{\eta_{2}}{2} \sinh \frac{\eta_{1}}{2} B_{1}^{0}=\cosh \frac{\alpha}{2} \sinh \frac{\eta}{2} \cos \frac{\theta}{2} B^{0} \\
& +\sinh \frac{\eta}{2} \sinh \frac{\alpha}{2}\left(\cos \frac{\theta}{2} \vec{B} \cdot \vec{b}+\sin \frac{\theta}{2} \epsilon_{j k i} r^{i} B^{j} b^{k}\right) \\
& \cosh \frac{\eta_{1}}{2} \sinh \frac{\eta_{2}}{2} B_{2}^{i}+\cosh \frac{\eta_{2}}{2} \sinh \frac{\eta_{1}}{2} B_{1}^{i}
\end{aligned}
$$




$$
\begin{aligned}
= & \cosh \frac{\alpha}{2} \sinh \frac{\eta}{2}\left(\cos \frac{\theta}{2} B^{i}-\sin \frac{\theta}{2} \epsilon_{m k}^{i} r^{m} B^{k}\right) \\
& +B^{0} \sinh \frac{\alpha}{2} \sinh \frac{\eta}{2}\left(\cos \frac{\theta}{2} b^{i}-\sin \frac{\theta}{2} b_{k} r_{j} \epsilon_{k j}^{i}\right)
\end{aligned}
$$

The other equations state that we have $\vec{r} \cdot \vec{b}=0$ and $\vec{r} \cdot \vec{B}=0$. This all together allows to reconstruct the sum of momenta given the choice of coordinates $k_{\mu}=\kappa f(\eta) B_{\mu}$. we are going to determine this sum in the Snyder's choice, i.e. $p_{\mu}=\kappa \tanh \eta B_{\mu}$. With this choice, we have in particular $\cosh \eta \equiv \Gamma=\frac{1}{\sqrt{1+\frac{p^{2}}{\kappa^{2}}}}$. Considering the ratio of the equation (A.2) with (A.1), together with $\tanh \frac{\eta}{2}=\frac{\tanh \eta}{1+\Gamma^{-1}}$, we have

$$
\tan \frac{\theta}{2} \vec{r}=\frac{\vec{p}_{1} \wedge \vec{p}_{2}}{\kappa^{2}\left(1+\Gamma_{1}^{-1}\right)\left(1+\Gamma_{2}^{-1}\right)-p_{1} \cdot p_{2}} \Leftrightarrow \vec{\rho}=\vec{A}\left(p_{1}, p_{2}\right),
$$

with $\vec{\rho}=\tan \frac{\theta}{2} \vec{r}$. Since $\vec{r}^{2}=1$, we have in particular that

$$
\tan ^{2} \frac{\theta}{2}=\left|\vec{A}\left(p_{1}, p_{2}\right)\right|^{2} \text {. }
$$

Considering the ratio of the equation (A.3) with (A.1), we obtain

$$
\begin{aligned}
\tanh & \frac{\alpha}{2}\left(\vec{b}-\tan \frac{\theta}{2} \vec{r} \wedge \vec{b}\right)=\frac{p_{1}^{0} \vec{p}_{2}-p_{2}^{0} \vec{p}_{1}}{\kappa^{2}\left(1+\Gamma_{1}^{-1}\right)\left(1+\Gamma_{2}^{-1}\right)-p_{1} \cdot p_{2}} \\
& \Leftrightarrow \tanh \frac{\alpha}{2}\left(\mathbb{1}-\tan \frac{\theta}{2} \vec{r} \wedge\right)(\vec{b})=\frac{p_{1}^{0} \vec{p}_{2}-p_{2}^{0} \vec{p}_{1}}{\kappa^{2}\left(1+\Gamma_{1}^{-1}\right)\left(1+\Gamma_{2}^{-1}\right)-p_{1} \cdot p_{2}} \\
& \Leftrightarrow \vec{F}(\vec{\beta})=\vec{C}\left(p_{1}, p_{2}\right),
\end{aligned}
$$

with $\vec{\beta}=\tanh \frac{\alpha}{2} \vec{b}$. The map $\vec{F}$ is a linear map and bearing in mind that $\vec{r} \cdot b=0$, its inverse $\vec{F}^{-1}$ is given by

$$
\vec{F}^{-1}=\cos ^{2} \frac{\theta}{2}\left(\mathbb{1}+\tan \frac{\theta}{2} \vec{r} \wedge\right)=\cos ^{2} \frac{\theta}{2} \vec{D},
$$

and is also a linear map. We can therefore define $\vec{\beta}=\tanh \frac{\alpha}{2} \vec{b}$ in terms of $p_{1}$ and $p_{2}$ since

$$
\vec{\beta}=\cos ^{2} \frac{\theta}{2} \vec{D}\left(\vec{C}\left(p_{1}, p_{2}\right)\right)=\cos ^{2} \frac{\theta}{2}(\mathbb{1}+\vec{\rho} \wedge)\left(\frac{p_{1}^{0} \vec{p}_{2}-p_{2}^{0} \vec{p}_{1}}{\kappa^{2}\left(1+\Gamma_{1}^{-1}\right)\left(1+\Gamma_{2}^{-1}\right)-p_{1} \cdot p_{2}}\right) .
$$

Now since $\cos ^{2} \frac{\theta}{2}=\left(1+\tan ^{2} \frac{\theta}{2}\right)^{-1}$ and $\tan ^{2} \frac{\theta}{2}$ is given in (A.6), we can determine $\cos ^{2} \frac{\theta}{2}$ in terms of $A\left(p_{1}, p_{2}\right)$ and hence $\vec{\beta}$ in terms of $p_{1}$ and $p_{2}$.

Considering the ratio of the equation (A.5) with (A.1), we obtain

$$
\begin{aligned}
& \kappa\left(\frac{\left(1+\Gamma_{2}^{-1}\right) \vec{p}_{1}+\left(1+\Gamma_{1}^{-1}\right) \vec{p}_{2}}{\kappa^{2}\left(1+\Gamma_{1}^{-1}\right)\left(1+\Gamma_{2}^{-1}\right)-p_{1} \cdot p_{2}}\right)=\tanh \frac{\eta}{2}(\vec{B}-\vec{\rho} \wedge \vec{B})+\tanh \frac{\eta}{2} B^{0}(\vec{\beta}+\vec{\rho} \wedge \vec{\beta}), \\
& \vec{E}\left(p_{1}, p_{2}\right)=\tanh \frac{\eta}{2} \vec{F}(\vec{B})+\tanh \frac{\eta}{2} B^{0} \vec{D}(\vec{\beta}) .
\end{aligned}
$$

We can use the inverse map $\vec{F}^{-1}=\cos ^{2} \frac{\theta}{2} \vec{D}$ to determine $\vec{B}$ in terms of $B_{0}, p_{1}, p_{2}$ and $\eta$, since we have that $\vec{r} \cdot \vec{B}=0$,

$$
\tanh \frac{\eta}{2} \vec{B}=-\tanh \frac{\eta}{2} B^{0} \cos ^{2} \frac{\theta}{2} \vec{D}(\vec{D}(\vec{\beta}))+\cos ^{2} \frac{\theta}{2} \vec{D}\left(\vec{E}\left(p_{1}, p_{2}\right)\right)
$$




$$
\Leftrightarrow \tanh \frac{\eta}{2} \vec{B}=-\tanh \frac{\eta}{2} B^{0} \vec{V}+\vec{W}
$$

where $\vec{V} \equiv \cos ^{2} \frac{\theta}{2} \vec{D}(\vec{D}(\vec{\beta}))$ and $\vec{W} \equiv \cos ^{2} \frac{\theta}{2} \vec{D}\left(\vec{E}\left(p_{1}, p_{2}\right)\right)$ are vectors totally determined in terms of $p_{1}$ and $p_{2}$.

Considering the ratio of the equation (A.4) with (A.1), we obtain

$$
\begin{aligned}
& \kappa\left(\frac{\left(1+\Gamma_{2}^{-1}\right) p_{1}^{0}+\left(1+\Gamma_{1}^{-1}\right) p_{2}^{0}}{\kappa^{2}\left(1+\Gamma_{1}^{-1}\right)\left(1+\Gamma_{2}^{-1}\right)-p_{1} \cdot p_{2}}\right)=\tanh \frac{\eta}{2}\left(B^{0}+(\vec{B} \cdot \vec{\beta}+\vec{B} \cdot(\vec{\rho} \wedge \vec{\beta}))\right) \\
& \Leftrightarrow I\left(p_{1}, p_{2}\right)=\tanh \frac{\eta}{2}\left(B^{0}+\vec{B} \cdot \vec{D}(\vec{\beta})\right) .
\end{aligned}
$$

We can used now the value for $\vec{B}$ identified in (A.7)

$$
\begin{aligned}
\tanh & \frac{\eta}{2} B^{0}+\left(-\tanh \frac{\eta}{2} B^{0} \vec{V}+\vec{W}\right) \cdot \vec{D}(\vec{\beta})=I\left(p_{1}, p_{2}\right) \\
& \Leftrightarrow \tanh \frac{\eta}{2} B^{0}(1-\vec{V} \cdot \vec{D}(\vec{\beta}))=-\vec{W} \cdot \vec{D}(\vec{\beta})+I\left(p_{1}, p_{2}\right) \\
& \Leftrightarrow \tanh \frac{\eta}{2} B^{0}=\frac{-\vec{W} \cdot \vec{D}(\vec{\beta})+I\left(p_{1}, p_{2}\right)}{1-\vec{V} \cdot \vec{D}(\vec{\beta})} .
\end{aligned}
$$

We can then plug the value of $B_{0}$ into (A.7) to obtain $B_{i}$ in terms of the $p_{i}$

$$
\tanh \frac{\eta}{2} \vec{B}=\frac{-\vec{W} \cdot \vec{D}(\vec{\beta})+I\left(p_{1}, p_{2}\right)}{1-\vec{V} \cdot \vec{D}(\vec{\beta})} \vec{V}
$$

The final step to recover the sum is to recall that $\tanh \frac{\eta}{2}=\frac{\tanh \eta}{1+\Gamma^{-1}}$ and $\Gamma$ can be expressed in terms of $\Gamma_{i}$

$$
\Gamma=\Gamma_{1} \Gamma_{2}\left(1-\frac{p_{1} \cdot p_{2}}{\kappa^{2}}\right) .
$$

Hence we have that

$$
\begin{aligned}
& \vec{p}_{\text {tot }}=\overrightarrow{p_{1} \oplus p_{2}}=\left(1+\Gamma_{1}^{-1} \Gamma_{2}^{-1}\left(1-\frac{p_{1} \cdot p_{2}}{\kappa^{2}}\right)^{-1}\right)\left(\frac{-\vec{W} \cdot \vec{D}(\vec{\beta})+I\left(p_{1}, p_{2}\right)}{1-\vec{V} \cdot \vec{D}(\vec{\beta})}+\vec{W}\right), \\
& p_{\text {tot }}^{0}=\left(p_{1} \oplus p_{2}\right)^{0}=\left(1+\Gamma_{1}^{-1} \Gamma_{2}^{-1}\left(1-\frac{p_{1} \cdot p_{2}}{\kappa^{2}}\right)^{-1}\right) \frac{-\vec{W} \cdot \vec{D}(\vec{\beta})+I\left(p_{1}, p_{2}\right)}{1-\vec{V} \cdot \vec{D}(\vec{\beta})} .
\end{aligned}
$$

\section{References}

[1] Snyder H., Quantized space-time, Phys. Rev. 71 (1947), 38-41.

[2] Breckenridge J.C., Elias V., Steele T.G., Massless scalar field theory in a quantised spacetime, Classical Quantum Gravity 12 (1995), 637-650, hep-th/9501108.

[3] Girelli F., Livine E.R., Field theories with homogenous momentum space, in Proceedings of 25th Max Born Symposium: The Planck Scale (Wroclaw, Poland, 2009), AIP Conf. Proc. 1196, (2009), 115-123, arXiv:0910.3107.

[4] Girelli F., Livine E.R., Scalar field theory in Snyder space-time: alternatives, arXiv:1004.0621.

[5] Battisti M.V., Meljanac S., Scalar field theory on non-commutative Snyder space-time, Phys. Rev. D 82 (2010), 024028, 9 pages, arXiv:1003.2108.

Meljanac S., Meljanac D., Samsarov A., Stojic M., $\kappa$-deformed Snyder spacetime, Modern Phys. Lett. A 25 (2010), 579-590, arXiv:0912.5087.

Battisti M.V., Meljanac S., Modification of Heisenberg uncertainty relations in non-commutative Snyder space-time geometry, Phys. Rev. D 79 (2009), 067505, 4 pages, arXiv:0812.3755. 
[6] Zakrzewski S., Poisson structures on the Poincaré group, Comm. Math. Phys. 185 (1997), 285-311, q-alg/9602001.

[7] Lukierski J., Ruegg H., Nowicki A., Tolstoi V.N., q-deformation of Poincaré algebra, Phys. Lett. B 264 (1991), 331-338.

[8] Majid S., Ruegg H., Bicrossproduct structure of $\kappa$-Poincaré group and noncommutative geometry, Phys. Lett. B 334 (1994), 348-354, hep-th/9405107.

[9] Majid S., Foundations of quantum group theory, Cambridge University Press, Cambridge, 1995.

[10] Kowalski-Glikman J., Nowak S., Quantum $\kappa$-Poincaré algebra from de Sitter space of momenta, hep-th/0411154.

[11] Freidel L., Kowalski-Glikman J., Nowak S., Field theory on $\kappa$-Minkowski space revisited: Noether charges and breaking of Lorentz symmetry, Internat. J. Modern Phys. A 23 (2008), 2687-2718, arXiv:0706.3658.

[12] Kiechle H., Theory of K-loops, Lecture Notes in Mathematics, Vol. 1778, Springer-Verlag, Berlin, 2002.

[13] Joung E., Mourad J., Noui K., Three dimensional quantum geometry and deformed symmetry, J. Math. Phys. 50 (2009), 052503, 29 pages, arXiv:0806.4121.

[14] Sabinin L.V., Smooth quasigroups and loops, Mathematics and Its Applications, Vol. 492, Kluwer Academic Publishers, Dordrecht, 1999.

[15] Klim J., Majid S., Hopf quasigroups and the algebraic 7-sphere, J. Algebra 323 (2010), 3067-3110, arXiv:0906.5026.

Klim J., Majid S., Bicrossproduct Hopf quasigroups, arXiv:0911.3114.

[16] Kikkawa M., Geometry of homogeneous Lie loops, Hiroshima Math. J. 5 (1975), 141-179.

[17] Ungar A.A., Thomas precession and its associated grouplike structure, Amer. J. Phys. 59 (1991), 824-834.

[18] Sabinin L.V., Sabinina L.L., Sbitneva L.V., On the notion of gyrogroup, Aequationes Math. 56 (1998), 11-17.

[19] Girelli F., Livine E.R., Special relativity as a noncommutative geometry: lessons for deformed special relativity, Phys. Rev. D 81 (2010), 085041, 17 pages, gr-qc/0407098.

[20] Mostovoy J., Perez-Izquierdo J.M., Ideals in non-associative universal enveloping algebras of Lie triple systems, math/0506179.

Perez-Izquierdo J.M., Algebras, hyperalgebras, nonassociative bialgebras and loops, Adv. Math. 208 (2007), 834-876.

[21] Nagy G.P., The Campbell-Hausdorff series of local analytic Bruck loops, Abh. Math. Sem. Univ. Hamburg 72 (2002), 79-87.

[22] Jacobson N., General representation theory of Jordan algebras, Trans. Amer. Math. Soc. 70 (1951), 509-530.

[23] Hodge T.L., Parshall B.J., On the representation theory of Lie triple systems, Trans. Amer. Math. Soc. 354 (2002), 4359-4391.

[24] Lister W.G., A structure theory of Lie triple systems, Trans. Amer. Math. Soc. 72 (1952), $217-242$.

[25] Doplicher S., Fredenhagen K., Roberts J.E., The quantum structure of spacetime at the Planck scale and quantum fields, Comm. Math. Phys. 172 (1995), 187-220, hep-th/0303037.

[26] Carlson C.E., Carone C.D., Zobin N., Noncommutative gauge theory without Lorentz violation, Phys. Rev. D 66 (2002), 075001, 8 pages, hep-th/0206035.

[27] Sitarz A., Noncommutative differential calculus on the $\kappa$-Minkowski space, Phys. Lett. B 349 (1995), 42-48, hep-th/9409014.

[28] Girelli F., Livine E.R., Physics of deformed special relativity, gr-qc/0412079.

Girelli F., Livine E.R., Physics of deformed special relativity: relativity principle revisited, Braz. J. Phys. 35 (2005), 432-438, gr-qc/0412004.

Andriot D., Lorentz precession in deformed special relativity, unpublished work. 